\title{
Melanomas with activating RAF1 fusions: clinical, histopathologic, and molecular profiles
}

\author{
Erik A. Williams $\mathbb{1}^{1} \cdot$ Nikunj Shah ${ }^{1} \cdot$ Meagan Montesion $\mathbb{1}^{1} \cdot$ Radwa Sharaf $^{1} \cdot$ Dean C. Pavlick $^{1} \cdot$ Ethan S. Sokol $^{1}$. \\ Brian M. Alexander ${ }^{1} \cdot$ Jeff M. Venstrom ${ }^{1}$. Julia A. Elvin ${ }^{1} \cdot$ Jeffrey S. Ross ${ }^{1,2} \cdot$ Julie Y. Tse $^{1,3} \cdot$ Mark C. Mochel $^{4}$
}

Received: 29 November 2019 / Revised: 19 February 2020 / Accepted: 19 February 2020 / Published online: 2 March 2020

(c) The Author(s) 2020. This article is published with open access

\begin{abstract}
A subset of melanomas is characterized by fusions involving genes that encode kinases. Melanomas with RAF1 fusions have been rarely reported, mostly in clinical literature. To investigate this distinctive group of melanomas, we searched for melanomas with activating structural variants in $R A F 1$, utilizing our case archive of clinical samples with comprehensive genomic profiling (CGP) by a hybrid capture-based DNA sequencing platform. Clinical data, pathology reports, and histopathology were reviewed for each case. RAFl breakpoints, fusion partners, and co-occurring genetic alterations were characterized. From a cohort of 7119 melanomas, 40 cases $(0.6 \%)$ featured fusions that created activating structural variants in RAF1. Cases with activating RAF1 fusions had median age of 62 years, were $58 \%$ male, and consisted of 9 primary tumors and 31 metastases. Thirty-nine cases were cutaneous primary, while one case was mucosal (anal) primary. Primary cutaneous melanomas showed variable architectures, including wedge-shaped and nodular growth patterns. Cytomorphology was predominantly epithelioid, with only one case, a desmoplastic melanoma, consisting predominantly of spindle cells. $R A F 15^{\prime}$ rearrangement partners were predominantly intrachromosomal $(n=18)$, and recurrent partners included MAP4 $(n=3)$, CTNNA1 $(n=2), \operatorname{LRCH3}(n=2), \operatorname{GOLGA4}(n=2), \operatorname{CTDSPL}(n=2)$, and PRKAR2A $(n=2)$, all $5^{\prime}$ of the region encoding the kinase domain. RAFl breakpoints occurred in intron $7(n=32)$, intron $9(n=4)$, intron $5(n=2)$, and intron 6 $(n=2)$. Ninety-eight percent $(n=39)$ were wild type for $B R A F, N R A S$, and NF1 genomic alterations (triple wild type). Activating RAFl fusions were present in $2.1 \%$ of triple wild-type melanomas overall (39/1882). In melanomas with activating RAF1 fusions, frequently mutated genes included TERTp (62\%), CDKN2A (60\%), TP53 (13\%), ARID2 (10\%), and PTEN (10\%). Activating RAF1 fusions characterize a significant subset of triple wild-type melanoma (2.1\%) with frequent accompanying mutations in TERTp and CDKN2A. CGP of melanomas may improve tumor classification and inform potential therapeutic options, such as consideration of specific kinase inhibitors.
\end{abstract}

Supplementary information The online version of this article (https:// doi.org/10.1038/s41379-020-0510-7) contains supplementary material, which is available to authorized users.

Erik A. Williams

erwilliams@foundationmedicine.com

1 Foundation Medicine, Inc., 150 Second Street, Cambridge, MA 02141, USA

2 Department of Pathology, State University of New York Upstate Medical University, 766 Irving Avenue, Syracuse, NY 13210, USA

3 Department of Pathology \& Laboratory Medicine, Tufts University School of Medicine, 145 Harrison Ave, Boston, MA 02111, USA

4 Departments of Pathology and Dermatology, Virginia Commonwealth University School of Medicine, 1200 East Marshall Street, Richmond, VA 23298, USA

\section{Introduction}

The majority of melanomas harbor point mutations of $B R A F, N R A S, K I T$, or NF1 that drive tumor growth [1,2]. Kinase rearrangements, although less common, represent the oncogenic drivers in emerging subgroups of melanoma, often through activation of MAP kinase pathways. Rearrangements in several genes, including BRAF, RET, ROS1, $A L K, N T R K 1$, and NTRK3, have been characterized in subsets of melanoma [2-5]. Surprisingly, despite the central role of RAF1 in the MAP kinase pathway, there are only isolated reports of RAF1 (CRAF) fusions in melanomas, and the histopathologic characterizations of these tumors have been limited [6-8].

The literature on melanoma with rearrangements in kinase genes other than $R A F 1$ is extensive. In particular, 
many of these rearrangements are associated with melanomas that demonstrate characteristic spitzoid cytomorphology, leading to their classification as Spitz melanomas [9-11]. As specified in the most recent World Health Organization (WHO) classification [12], the term "Spitz melanoma" refers specifically to melanoma with both histologic changes reminiscent of Spitz nevi and a known oncogenic fusion driver, particularly of kinase-encoding genes. In contrast, "spitzoid melanoma" refers to melanoma with some morphologic resemblance to Spitz nevus, but without a known fusion driver.

Retrospective studies of spitzoid neoplasms have found activating fusions involving $B R A F, R E T, R O S 1, A L K$, or NTRK1 in $39 \%$ of melanomas with spitzoid morphology and just over 50\% of Spitz nevi and atypical Spitz tumors $[11,13]$. In the largest reported series to date, patients with fusion-positive Spitz melanomas showed a broad age distribution, ranging from 6 to 73 years old, with a median age of 31 years [11]. While Spitz nevi and atypical Spitz tumors with NTRK3 [14] and NTRK1 [15, 16] fusions, as well as pigmented spindle cell nevi of Reed with NTRK3 fusions [17], have shown distinctive clinical and histopathologic profiles, Spitz melanomas with these alterations generally do not show similarly distinguishing characteristics. Small series have characterized adult cutaneous melanomas with NTRK fusions, correlated with large epithelioid and amelanotic cytomorphology, and $B R A F$ fusions, which have been variably correlated with spitzoid morphology [3, 4, 18, 19]. A recent study described pediatric Spitz melanomas with MAP3K8 fusions or truncations which tended to show expansile growth, hypercellularity, deep mitoses, and ulceration [20].

Following the identification in our archive of an activating $R A F 1$-fusion melanoma that responded to therapy with a MEK inhibitor [21], we performed a search of our archive of 276,645 clinical samples to identify melanoma cases with $R A F 1$ fusions that created known or likely activating structural variants in $R A F 1$, defined as loss of the autoinhibitory domain but retention of the kinase domain. In this study, we present the first series of activating RAFIfusion melanomas with clinical-pathologic correlation, detailed descriptions of several new fusion variants, and a thorough characterization of accompanying mutations.

\section{Materials and methods}

\section{Cohort and genomic analyses}

Comprehensive genomic profiling (CGP) was performed in a Clinical Laboratory Improvement Amendments certified, College of American Pathologists-accredited laboratory (Foundation Medicine, Inc., Cambridge, MA, USA).
Approval for this study, including a waiver of informed consent and a HIPAA waiver of authorization, was obtained from the Western Institutional Review Board (Protocol No. 20152817). For quality assurance, the presence of diagnostic tumor tissue was confirmed on routine hematoxylin and eosin (H\&E)-stained slides before DNA extraction. In brief, $\geq 60 \mathrm{ng}$ of DNA was extracted from $40 \mu \mathrm{m}$ sections of 7119 melanoma specimens, in formalin fixed, paraffinembedded tissue blocks. The samples were assayed by CGP using adaptor ligation, and hybrid capture was performed for all coding exons from 287 (version 1) to 315 (version 2) cancer-related genes plus select introns from 19 (version 1) to 28 (version 2) genes frequently rearranged in cancer (Supplementary Table 1). Sequences were analyzed for all classes of genomic alterations, including short variant alterations (base substitutions, insertions, and deletions), copy number alterations (focal amplifications and homozygous deletions), and select gene fusions or rearrangements, by methods previously described [22-24]. Tumor mutational burden (TMB, mutations/Mb) was determined on $0.8-1.1 \mathrm{Mbp}$ of sequenced DNA [24]. Microsatellite instability was determined on up to 114 loci [25].

\section{Mutational signatures}

Mutational signatures were evaluated for all samples containing at least 20 nondriver somatic missense alterations. Signatures were given by analysis of the trinucleotide context and profiled using the Sanger COSMIC signatures of mutational processes in human cancer [26]. A positive signature was determined if a sample had at least a $40 \%$ fit to a mutational process [26]. The COSMIC UV signature is dominated by $\mathrm{C}>\mathrm{T}$ transition mutations in a $\mathrm{CC}$ or TT dinucleotide setting [27].

\section{Clinical-pathological analysis of melanoma cohort harboring activating RAF1 fusions}

The cohort of melanomas harboring activating RAFl fusions comprised 40 cases, each from a different patient. Assays with CGP (Foundation Medicine Cambridge, MA, USA) occurred during clinical care at other institutions. Clinicopathological data including patient age, gender, tumor site, tumor diameter, and stage were extracted from the accompanying pathology reports.

$\mathrm{H} \& \mathrm{E}$ stained sections from each of the 40 cases were assessed retrospectively by two board-certified dermatopathologists (JYT and MCM). Histologic parameters assessed on primary tumors included tumor silhouette (dome shape, plaque-like growth, nodular growth, etc.), symmetry, shape of the tumor base (wedge-shaped, flat, bulbous, etc.), presence of epidermal involvement (and intraepidermal growth patterns), ulceration, maturation, 
deep nested growth, fascicular growth, associated dermal fibrosis, Breslow depth, mitotic rate, grade of solar elastosis [28], and tumor-infiltrating lymphocytes. Cytologic features, assessed on all cases, included predominant cytomorphology (epithelioid, spindled, mixed epithelioid and spindled), cytoplasmic color and abundance, and nuclear features of chromatin quality, nucleolar prominence, and degree of pleomorphism.

The H\&E slides were independently diagnostic of melanoma for primary tumors and for pigmented metastases. In contrast, H\&E slides were not independently diagnostic for cases of metastatic melanoma that showed nonpigmented malignant epithelioid proliferations. Those cases required diagnostic corroboration with accompanying pathology reports for relevant historical and immunohistochemical details (e.g., documented Melan-A and S100 positivity to confirm the diagnosis of melanoma vs. other malignant epithelioid tumors).

Quantitative data were analyzed using the Fisher exact test owing to the categorical quality of the data and the size of the cohort. For TMB comparison between two groups, the nonparametric Mann-Whitney $U$ test was used. A twotailed $P$ value of $<0.05$ was considered to be statistically significant.

\section{Results}

\section{Clinical-pathologic features}

From an internal series of 7119 melanomas that had undergone prior hybrid capture-based DNA sequencing, 40 cases $(0.6 \%)$, each from a different patient, featured gene rearrangements that created known or likely activating structural variants in $R A F 1$, defined as loss of the autoinhibitory domain but retention of the kinase domain.

Among patients with activating $R A F 1$-rearranged melanomas, the ages ranged from 34 to 86 years, with a median of 62 years. There were 23 males and 17 females. All patients had clinically advanced disease. Clinical staging ranged from at least stage $2 \mathrm{~A}$ to stage 4 , with the majority of cases documented at stage $4(n=25$ of $40 ; 63 \%)$ and most of the remaining cases at either stage $3 \mathrm{~A}$ or $3 \mathrm{~B}(n=10$ of $40 ; 25 \%)$. Sequencing was performed on the original primary tumor in 8 primary cutaneous melanomas and on 31 metastatic disease samples. Of the metastatic samples, sites included regional lymph nodes $(n=8)$, in-transit metastasis $(n=1)$, and distant lymph nodes $(n=3)$. Additional distant metastatic sites included skin (subcutaneous $(n=4)$ and dermal $(n=2)$ ), soft tissue $(n=3)$, brain $(n=2)$, lung $(n=2)$, and one each involving liver, omentum, small intestine, adrenal, bone, and spleen. Thirty-nine cases were consistent with either primary cutaneous melanoma or metastatic melanoma from a skin primary, while one case was a primary melanoma of anal mucosa.

Primary cutaneous tumors occurred on the extremities and trunk showed a mean tumor diameter of $16 \mathrm{~mm}$ (range 3-40 mm), mean thickness of $6.6 \mathrm{~mm}$ (range $2.3-17 \mathrm{~mm}$ ), and mean mitotic rate of 5.5 per $\mathrm{mm}^{2}$ (range $1-14$ per $\mathrm{mm}^{2}$ ) (Table 1). Melanomas were classified as nodular (3), superficial spreading (2), unclassified (2), and desmoplastic (1). The two melanomas with an unclassified subtype comprised one specimen of broadly ulcerated and deeply invasive melanoma without assessable lesional edges (case 1) and another specimen of residual melanoma deep to scar (case 4).

Histopathologic examination of the eight primary cutaneous melanomas revealed heterogeneous features (Fig. 1). Three cases showed domed surfaces with wedge-shaped bases (Fig. 1a-g), two showed nodular growth in the dermis and subcutis, one showed nodular and diffusely infiltrative growth in the dermis and subcutis, one appeared plaque like with an exophytic component (Fig. 1c), and the desmoplastic melanoma appeared as a haphazard spindle cell proliferation in the dermis and subcutis with fibrosis and lymphoid aggregates. Four cases were plainly asymmetric, while four were subtly asymmetric. Four cases showed epidermal involvement, with two demonstrating melanoma

Table 1 Clinical and pathologic features of primary $R A F 1$-fusion cutaneous melanomas.

\begin{tabular}{|c|c|c|c|c|c|c|c|c|c|}
\hline Case No. & Gender & Age (years) & Location & Diameter $(\mathrm{mm})$ & Type & Cytology & Thickness (mm) & Ulcer & TMR (\#/mm sq.) \\
\hline 1 & Male & 52 & Leg & 40 & Unclassified & Epithelioid & 17.0 & Present & 8 \\
\hline 2 & Female & 69 & Leg & 9 & Nodular & Epithelioid & 2.8 & Absent & 3 \\
\hline 3 & Female & 54 & Back & 13 & SSM & Epithelioid & 6.3 & Absent & 4 \\
\hline 4 & Female & 39 & Abdomen & 9 & Unclassified & Epithelioid & 5.0 & Absent & 14 \\
\hline 5 & Female & 74 & Arm & 23 & Desmoplastic & Spindled & 13.0 & Absent & 2 \\
\hline 6 & Female & 65 & Back & 15 & SSM & Epithelioid & 3.3 & Absent & 10 \\
\hline 7 & Female & 34 & Leg & 3 & Nodular & Epithelioid & 3.0 & Absent & 1 \\
\hline 8 & Male & 68 & Abdomen & 19 & Nodular & Epithelioid & 2.3 & Absent & 2 \\
\hline
\end{tabular}

TMR tumor mitotic rate, \# number, SSM superficial spreading melanoma. 


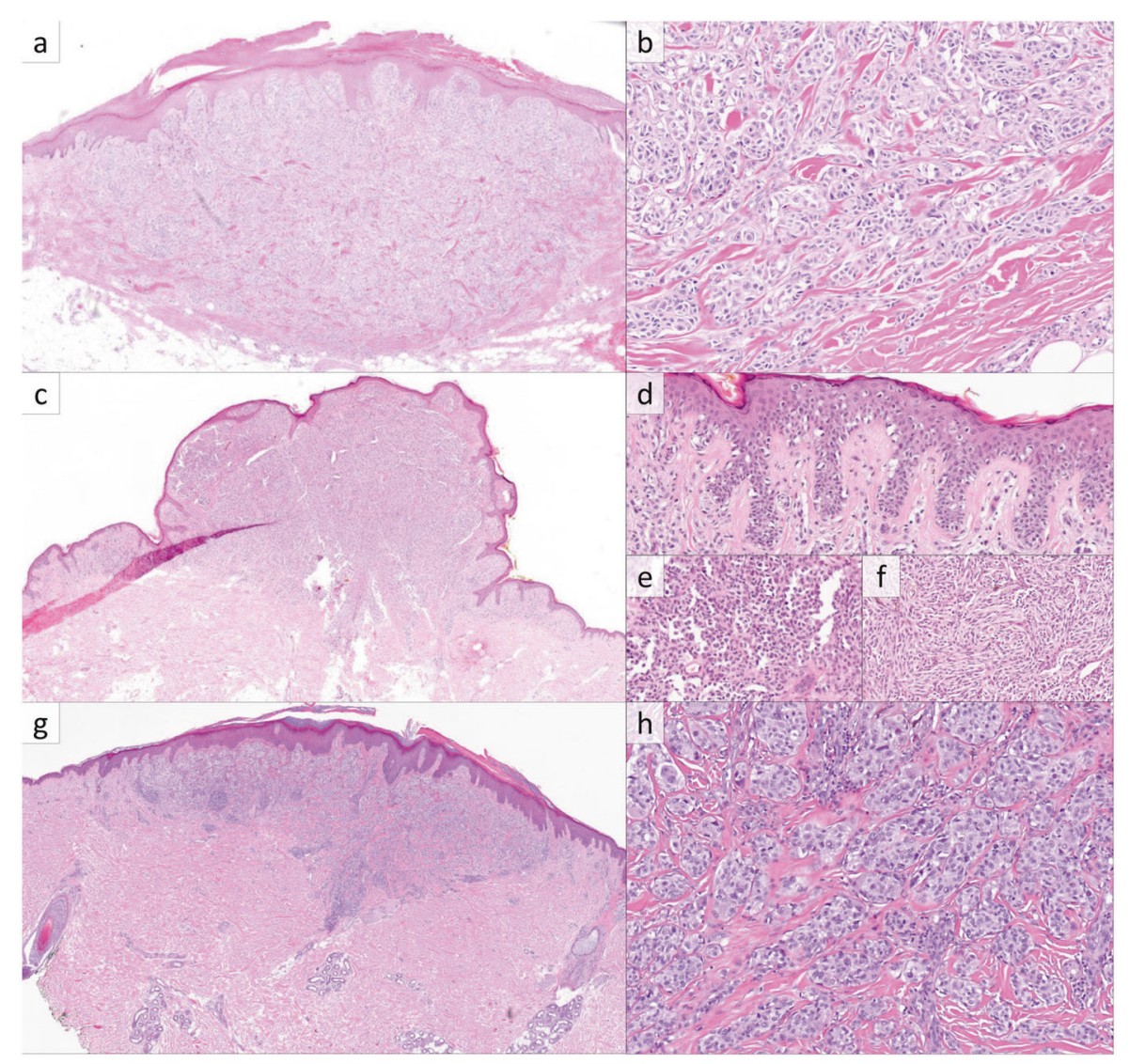

Fig. 1 RAF1-fusion melanoma, primary cutaneous lesions. a Histopathologic examination of case 2 reveals a melanocytic neoplasm with a slightly domed surface and wedge-shaped base centered in the dermis $(H \& E, \times 20)$. b The deep aspect of case 2 consists of nested large epithelioid melanocytes with associated dense collagen fibers and scattered mitotic figures $(\mathrm{H} \& \mathrm{E}, \times 200)$. c Case 3 showed an exophytic component with a plaque-like growth pattern at the periphery $(\mathrm{H} \& \mathrm{E}$, $\times 20$ ). For case 3 , the radial growth phase at the periphery showed intraepidermal growth with pagetoid scatter (d), while the vertical growth phase contained predominantly epithelioid melanocytes (e) with a deep zone of fascicular growth of spindled cells with focal cytoplasmic pigmentation (f) $(\mathrm{H} \& \mathrm{E}, \times 200, \times 400$, and $\times 400)$. $\mathbf{g}$, h Case 8 showed a melanocytic neoplasm with a domed surface and wedgeshaped base with nested growth in the deep aspect with dense collagen fibers and scattered mitoses (H\&E, $\times 20$ and $\times 200)$.

had grade 1, one had grade 2, and one could not be determined owing to lack of tumor-free and evaluable dermis in the H\&E sections. Tumor-infiltrating lymphocytes were absent or sparse in three cases, nonbrisk in four cases, and brisk in one case.

Among the eight cutaneous melanomas, melanocytic cytology was predominantly epithelioid in seven cases, and spindled in the desmoplastic melanoma case. Among the seven predominantly epithelioid cases, one showed focally spindled growth in the vertical growth phase (Fig. 1f). Their cytoplasm tended to be amphophilic (four cases) to palely eosinophilic (three cases), while one case had densely eosinophilic cytoplasm. Cytoplasmic quantity in the epithelioid cases was moderate to abundant in five and relatively scant in two. Only two showed cytoplasmic pigmentation (Fig. 1f), which was focal in both cases. Nuclear size was medium to large, and chromatin was heterogeneous (admixed dense and pale) in all cases. Nucleoli 
Fig. $2 R A F 1$-fusion melanoma, metastatic lesions. a Metastatic melanoma involving soft tissue with epithelioid cytomorphology (H\&E, ×400). b Metastatic melanoma involving liver with admixed epithelioid and spindled morphology, a pattern seen in only two metastases (H\&E, x400). c Metastatic melanoma involving a lymph node with eccentric dense eosinophilic cytoplasm, imparting a rhabdoid cytomorphology (H\&E, ×400). d Metastatic melanoma involving small intestine with large epithelioid cytology with cytoplasmic pigmentation (H\&E, ×400).

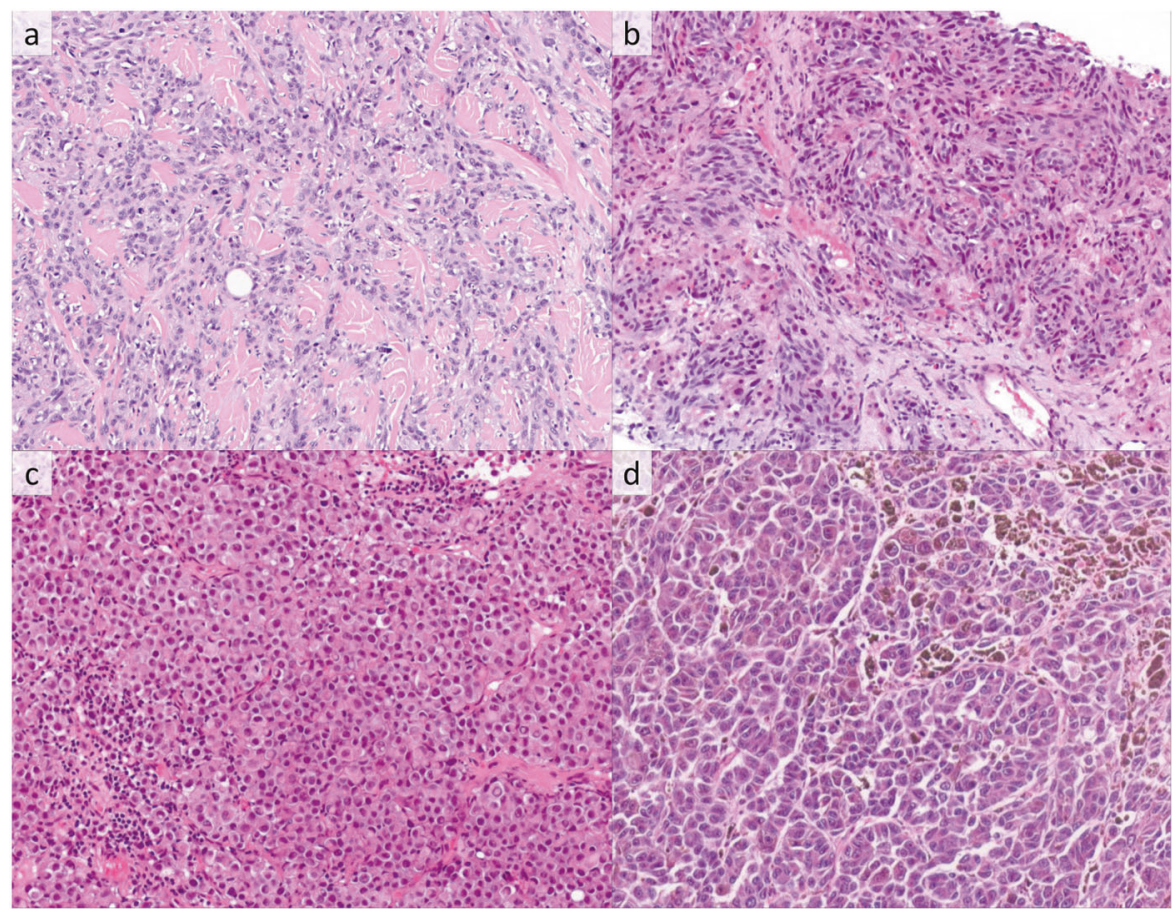

were prominent in three cases, small and distinct in two cases, and indistinct in three cases. Nuclear pleomorphism was judged to be severe in four cases and mild to moderate in the other four. No cases showed convincing Spitz-nevuslike cytomorphology (i.e., voluminous, homogeneous cytoplasm with sharp borders) or pulverocytic cytology characterized by pale, finely pigmented cytoplasm.

Among the 31 nodal and distant melanoma metastases, histopathology showed variable combinations of diffuse and nodular growth. While all metastatic tumors showed predominantly epithelioid cytomorphology (Fig. 2a), six (19\%) had eccentric dense eosinophilic cytoplasm paired with large nuclei with prominent nucleoli, imparting a rhabdoid appearance (Fig. 2c), and two (6\%) showed mixed epithelioid and spindled cytology (Fig. 2b). Cytoplasm was amphophilic (15 cases), palely eosinophilic (8 cases), and densely eosinophilic (8 cases). Only five metastatic cases showed cytoplasmic pigmentation, which was focal in three cases and diffuse in two (Fig. 2d). Nuclear size was medium to large in all cases, and nucleoli were prominent in 13 (42\%). Nuclear pleomorphism was severe in 16 cases (52\%) and mild-moderate in 15 cases $(48 \%)$.

\section{Comprehensive genomic profiling}

$5^{\prime}$ rearrangement partners were predominantly intrachromosomal $(n=17)$, and recurrent partners included MAP4 $(n=3)$, CTNNA1 $(n=2)$, LRCH3 $(n=2)$, GOLGA4 $(n=$ 2), CTDSPL $(n=2)$, and PRKAR2A $(n=2)$ (Fig. 3a). RAF1 breakpoints occurred in intron $7(n=32)$, intron $9(n=4)$, intron $5(n=2)$, and intron $6(n=2)$, i.e., all $5^{\prime}$ of the region encoding the kinase domain (Fig. 3a).

Of the activating $R A F 1$-fusion melanomas, $98 \%(n=39)$ were wild type for $B R A F, N R A S$, and NF1 genomic alterations (triple wild type) vs. $26 \%(n=1843 / 7079)$ of the melanoma cohort without RAF1 fusion ( $p<0.0001$, Fisher's exact test). The most frequently mutated other genes in $R A F 1$-fusion melanomas were TERTp (62\%; 23/37), CDKN2A (60\%; 24/40), TP53 (13\%; 5/40), SPTA (12\%; 3/26), FOXP1 (12\%; 4/27), ARID2 (10\%; 3/29), and PTEN (10\%; 4/40) (Fig. 3b). Median TMB was $10.2 \mathrm{mut} / \mathrm{Mb}$ (range $0-57.4,35 \% 10-20,18 \%>20$ ), similar to the primary skin and anus melanoma cohort without activating $R A F 1$ fusions (median TMB $=13.8,19 \% 10-20,36 \%>20$; $p=0.1$, Mann-Whitney $U$ test). Copy number plots showed complex gains and losses. All evaluated cases were microsatellite stable. Of the 28 cases with the requisite number of mutations for UV signature identification, 22 harbored a positive UV signature (79\%). Of the remaining six cases, three had a UV signature $<40 \%$ $(10,29$, and $38 \%)$.

Comparison of tumors sequenced from the primary sites, regional metastases, and distant metastases was performed. Tumors sequenced from primary sites vs. metastases showed similar percentages of genomic alterations, including in TERTp, CDKN2A, TP53, and PTEN (56\% vs. 64\%, $67 \%$ vs. $58 \%, 11 \%$ vs. $13 \%$, and $11 \%$ vs. $10 \%$, respectively). Similarly, no significant differences in percentages of genomic alterations were identified between tumors sequenced from regional vs. distant metastases. 
a

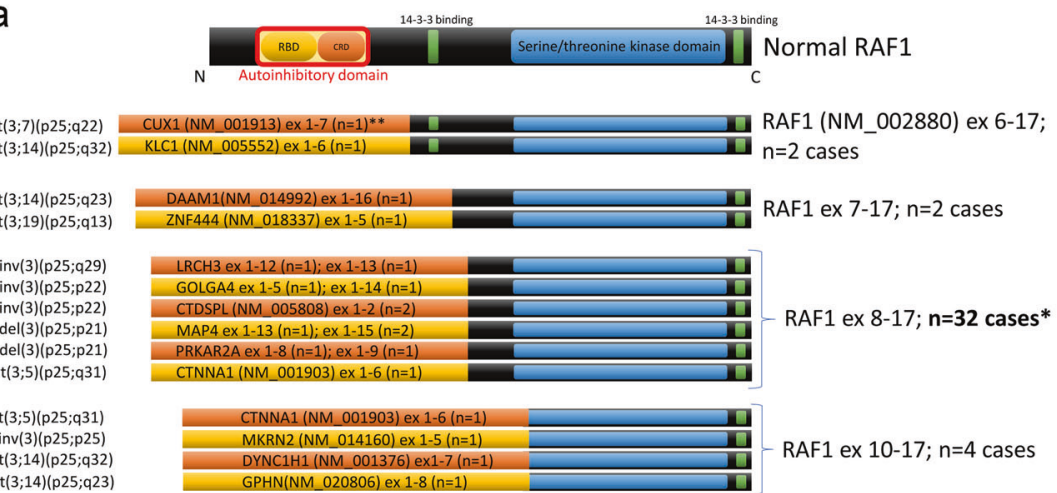

b

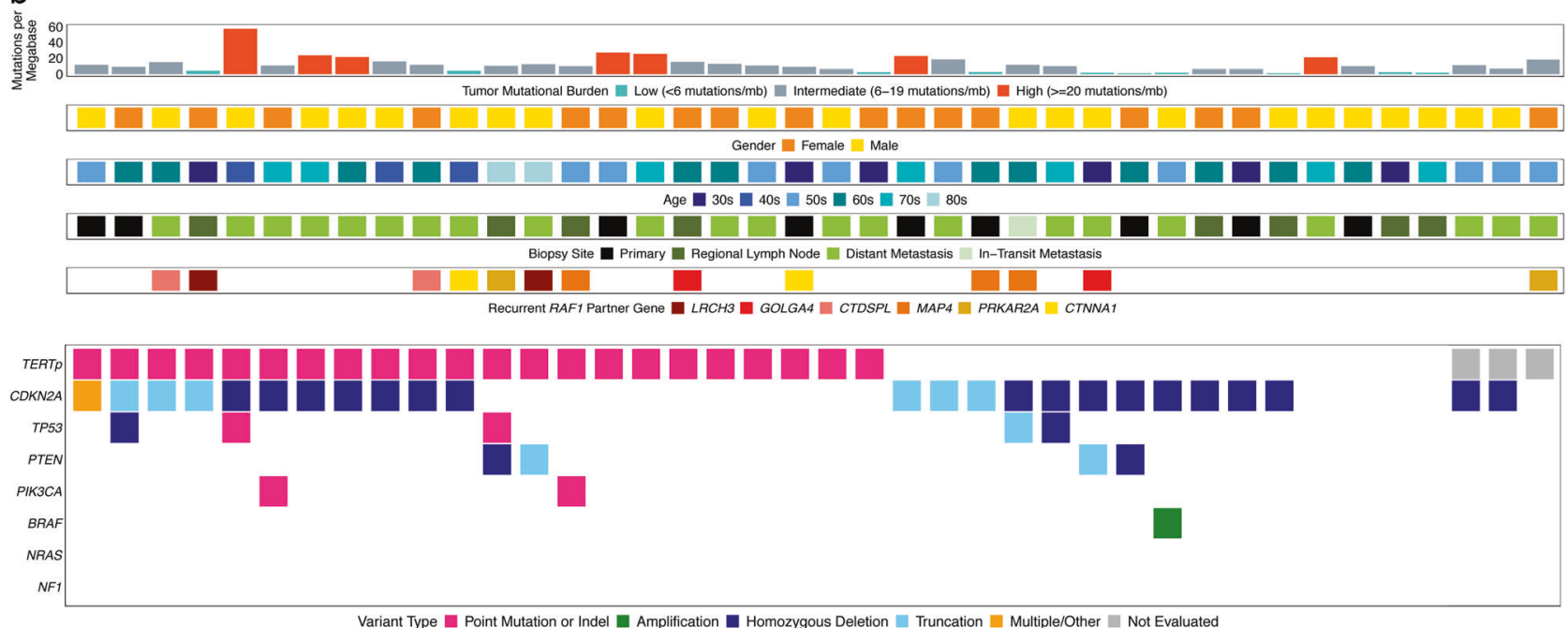

Fig. 3 Molecular profiles of activating RAF1-fusion melanomas. a Schematic of functional domains of RAF1 and structure of activating RAF1 fusions showing loss of the autoinhibitory domain but retention of the RAF1 kinase domain. Recurrent RAF1 breakpoints were identified in introns 5-7 and 9, i.e., $5^{\prime}$ of the region encoding the kinase

Three additional cases with $R A F 1$ rearrangements that did not result in loss of the regulatory domain of $R A F 1$ were identified, including two RAFl intergenic rearrangements (exon 2-6 inversion and exon 6-7 duplication) and one $O P R M 1-R A F 1$ rearrangement with $R A F 1$ breakpoint at exon 2. The exon inversion case was of vulvar origin with two copy loss of $C D K N 2 A$, while the remaining two cases were cutaneous in origin and had pathogenic $B R A F$ mutations.

\section{Discussion}

To our knowledge, this study represents the first series of activating $R A F 1$-fusion melanomas with clinicopathologic correlation and detailed characterization of genetic alterations. Activating $R A F 1$ fusions represent a significant subset of triple wild-type melanoma ( $2.1 \%$ of all triple wild-type domain. b Summary of clinical features and molecular alterations in activating $R A F 1$-fusion melanomas. RBD Ras-binding domain, CRD cysteine-rich domain, TERTp TERT promoter. *Of these 32 cases, only recurrent partners with RAF1 are depicted. $* * R A F 1$ partner exon size is not representative in any case.

melanoma). Recurrent fusion partners and recurrent $R A F 1$ breakpoints were present. Frequent accompanying mutations in TERTp and CDKN2A were identified, typical for skin primary melanoma, and TMB was not significantly different from primary skin and anus melanoma cases without activating $R A F 1$ fusions.

Reports of activating $R A F 1$ fusions in melanocytic neoplasms have been rare. In one study seeking therapeutically targetable gene fusions in multiple cancer types, FISH for $B R A F$ and $R A F 1$ performed on 131 melanomas identified one $B R A F$ rearrangement and one $R A F 1$ rearrangement [6]. A more recent study of kinase fusions across large numbers of various malignancies found four cases of $R A F 1$ fusion out of 397 melanomas [29]. A whole-genome study of 183 melanomas found $R A F 1$ fusions in two melanomas (with partners $C D H 3$ and GOLGA4): one triple wild type and one with an NF1 comutation [30]. In a study of 21 large to giant congenital nevi, one case was found to have a SOX5-RAFI 
fusion [31]. This congenital nevus, like that of an $A L K$ fused nevus in the same study, lacked comutations of NRAS and $B R A F$. A striking case report also showed a fusion of SASS6-RAF1 in a giant congenital nevus that gave rise to melanoma with rhabdomyosarcomatous differentiation [32]. While a balanced translocation was found in the background congenital nevus, an unbalanced translocation was noted in the rhabdomyosarcomatous component.

Of note, activating $R A F 1$ fusions are also found in a small proportion of thyroid carcinomas, prostatic adenocarcinomas, and pilocytic astrocytomas [29, 33, 34]. Atefi et al. described RAF1 missense point mutation $\mathrm{R} 391 \mathrm{~W}$ in one melanoma cell line that lacked common driver mutations and showed resistance to vemurafenib despite MAPK signaling [7]. One report described a melanoma with GOLGA4 fused to exons 8-17 of $R A F 1$, retaining the $R A F 1$ kinase domains, and with accompanying mutations in CTNNB1 and CDKN2A [8]. Importantly, this patient's melanoma showed a marked clinical response to therapeutic MEK inhibition, as indicated by serial PET scans.

Prior histopathologic descriptions of activating RAF1fusion melanomas are limited. We observed various architectural patterns, including wedge-shaped growth with associated epidermal hyperplasia and deep nested melanocytes in three cases, reminiscent of some Spitz tumors with $A L K$ and NTRK1 fusions [10]. In contrast to Spitz tumors with fusions of $A L K, N T R K 1$, and ROS1, which are usually compound with a prominent epidermal component, epidermal involvement in the $R A F 1$-fused melanomas was typically limited or absent $[9,10,16,35,36]$. Characteristic spitzoid cytomorphology was not observed. Rather, we noted a somewhat heterogeneous group of tumors with predominantly epithelioid melanocytic cytomorphology with amphophilic to eosinophilic cytoplasm and medium to large nuclei with heterogeneous chromatin and often prominent nucleoli. In some cases, the large epithelioid cytology resembled that reported in NTRK-fusion melanomas, as well as in some Spitz tumors with $A L K$ translocation $[4,13,36]$. In our cases of primary cutaneous melanoma with activating $R A F 1$ fusions, a distinctive growth pattern, such as the fascicular pattern of $A L K 1$-fused Spitz tumors, was not seen. Nevertheless, typical histopathologic features of melanoma, including asymmetry, lack of maturation, cytologic atypia, and dermal mitotic activity, were readily identifiable in the cases evaluated in our study.

Triple wild-type melanomas (BRAF, RAS, and NF1 wild type) typically lack a UV signature [37]. In our series, however, activating $R A F 1$-fusion melanoma was almost entirely triple wild type, cutaneous, and UV driven. Given the frequent concurrent mutations in TERTp, CDKN2A, and TP53, and the relatively low TMB $($ median $=10.2 \mathrm{mut} / \mathrm{Mb})$, these tumors may fit best into the low cumulative sun damage group, as described in the current WHO classification [28]. Concordant with this classification, primary cases in our cohort generally lacked significant solar elastosis.

The $R A F 1$ fusions we identified appear to be pathogenic, given that the most significant regulatory mechanism for RAF1 is the direct association of the N-terminal autoinhibitory domains to the kinase domain. Loss of this domain but retention of the kinase domains, as seen in each of our cases (Fig. 3a), would cause autonomous, unregulated activation of kinase activity [38].

Limitations of this study include its retrospective nature and the distinct population of patients highly enriched for aggressive tumors, mostly metastatic to distant sites. Typically, extensive genomic testing is performed on advanced malignancies from patients whose oncologists are seeking targeted therapies. Thus, this patient population may not be representative of the general population of patients with melanoma. We note that the median age of 62 years for $R A F 1$-fusion melanomas in this study is significantly older than that reported in prior melanocytic tumors with gene fusions, which tend toward pediatric and young adult patients. While an actual age difference may exist, this age discrepancy may be attributable to disparate study cohorts: our cohort was selected from melanomas with proven aggressive behavior, while other fusion-positive melanocytic tumor cohorts often were selected for Spitz morphology, often in diagnostically challenging cases, and therefore enriched for young patients. Finally, while our review of histologic slides from all cases enabled us to confirm histopathologic diagnoses, particularly for cases of primary cutaneous melanoma and pigmented metastatic lesions where H\&E slides were independently diagnostic, some cases required corroboration with details from the accompanying pathology reports (e.g., for metastatic melanomas with histologic slides showing malignant epithelioid proliferations, we relied on corresponding pathology reports for confirmatory immunohistochemical details).

CGP of melanomas may provide insights into pathogenesis, as well as potential therapeutic options. Additional studies will be needed to correlate the finding of activating $R A F 1$ fusions in melanoma with prognostic data and treatment outcomes. Prognostic data will also enable the comparison of $R A F 1$-fused melanomas by comutations, such as TERTp, which has been shown to be an important prognostic marker for spitzoid melanocytic neoplasms [39]. Furthermore, the spectrum of melanocytic lesions with activating $R A F 1$ rearrangements may be wider than the highly selected, aggressive tumors examined in this study. Overall, our findings provide a compelling rationale for consideration of CGP of melanomas, which may offer insights into melanoma biology and potentially inform therapeutic options, including specific kinase inhibitors. 


\section{Compliance with ethical standards}

Conflict of interest EAW, NS, MM, RS, DCP, ESS, BMA, JMV, JAE, JSR, and JYT are employees of Foundation Medicine, Inc., a wholly owned subsidiary of Roche Holdings, Inc. and Roche Finance Ltd, and these employees have equity interest in an affiliate of these Roche entities. MCM declares no conflict of interest.

Ethical approval IRB approval status: reviewed and approved by Western IRB; Protocol No. 20152817.

Publisher's note Springer Nature remains neutral with regard to jurisdictional claims in published maps and institutional affiliations.

Open Access This article is licensed under a Creative Commons Attribution 4.0 International License, which permits use, sharing, adaptation, distribution and reproduction in any medium or format, as long as you give appropriate credit to the original author(s) and the source, provide a link to the Creative Commons license, and indicate if changes were made. The images or other third party material in this article are included in the article's Creative Commons license, unless indicated otherwise in a credit line to the material. If material is not included in the article's Creative Commons license and your intended use is not permitted by statutory regulation or exceeds the permitted use, you will need to obtain permission directly from the copyright holder. To view a copy of this license, visit http://creativecommons. org/licenses/by/4.0/

\section{References}

1. Curtin JA, Fridlyand J, Kageshita T, Patel HN, Busam KJ, Kutzner H, et al. Distinct sets of genetic alterations in melanoma. N Engl J Med. 2005;353:2135-47.

2. Bastian BC. The molecular pathology of melanoma: an integrated taxonomy of melanocytic neoplasia. Annu Rev Pathol. 2014;9: 239-71.

3. Turner J, Couts K, Sheren J, Saichaemchan S, Ariyawutyakorn W, Avolio I, et al. Kinase gene fusions in defined subsets of melanoma. Pigment Cell Melanoma Res. 2017;30:53-62.

4. Lezcano C, Shoushtari AN, Ariyan C, Hollmann TJ, Busam KJ. Primary and metastatic melanoma with NTRK fusions. Am J Surg Pathol. 2018;42:1052-8.

5. Botton T, Yeh I, Bastian BC. Melanoma BRAF fusions-letter. Clin Cancer Res. 2014;20:6631.

6. Palanisamy N, Ateeq B, Kalyana-Sundaram S, Pflueger D, Ramnarayanan K, Shankar S, et al. Rearrangements of the RAF kinase pathway in prostate cancer, gastric cancer and melanoma. Nat Med. 2010;16:793-8.

7. Atefi M, Titz B, Tsoi J, Avramis E, Le A, Ng C, et al. CRAF R391W is a melanoma driver oncogene. Sci Rep. 2016; 6:1-11.

8. McEvoy CR, Xu H, Smith K, Etemadmoghadam D, Leong HS, Choong DY, et al. Profound MEK inhibitor response in a cutaneous melanoma harboring a GOLGA4-RAF1 fusion. J Clin Invest. 2019;129:1940-5.

9. Yeh I, De La Fouchardiere A, Pissaloux D, Mully TW, Garrido MC, Vemula SS, et al. Clinical, histopathologic, and genomic features of spitz tumors with ALK fusions. Am J Surg Pathol. 2015;39:581-91.

10. Amin SM, Haugh AM, Lee CY, Zhang B, Bubley JA, Merkel EA, et al. A comparison of morphologic and molecular features of BRAF, ALK, and NTRK1 fusion spitzoid neoplasms. Am J Surg Pathol. 2017;41:491-8.
11. Wiesner T, He J, Yelensky R, Esteve-Puig R, Botton T, Yeh I, et al. Kinase fusions are frequent in Spitz tumours and spitzoid melanomas. Nat Commun. 2014;5:3116.

12. Barnhill RL, Bahrami A, Bastian BC, Busam K, Cerroni L, de la Fouchardiere A, et al. Malignant Spitz tumour (Spitz melanoma). In: Elder D, Massi D, Scolyer R, et al., editors. World Health Organization 2018 Classification of Skin Tumours. Lyon, France: International Agency for Research on Cancer, 2018. p. 108-10.

13. Wiesner T, Kutzner H, Cerroni L, Mihm MC, Busam KJ, Murali R. Genomic aberrations in spitzoid melanocytic tumours and their implications for diagnosis, prognosis and therapy. Pathology. 2016;48:113-31.

14. Yeh I, Tee MK, Botton T, Shain AH, Sparatta AJ, Gagnon A, et al. NTRK3 kinase fusions in Spitz tumours. J Pathol. 2016; 240:282-90.

15. Yeh I, Busam KJ, McCalmont TH, LeBoit PE, Pissaloux D, Alberti L, et al. Filigree-like rete ridges, lobulated nests, rosettelike structures, and exaggerated maturation characterize Spitz tumors With NTRK1 fusion. Am J Surg Pathol. 2019;43:737-46.

16. Kiuru M, Jungbluth A, Kutzner H, Wiesner T, Busam KJ. Spitz tumors: comparison of histological features in relationship to immunohistochemical staining for ALK and NTRK1. Int J Surg Pathol. 2015;24:200-6.

17. Vandenboom T, Quan VL, Zhang B, Garfield EM, Kong BY, Isales $\mathrm{MC}$, et al. Genomic fusions in pigmented spindle cell nevus of reed. Am J Surg Pathol. 2018;42:1042-51.

18. Hutchinson KE, Lipson D, Stephens PJ, Otto G, Lehmann BD, Lyle PL, et al. BRAF fusions define a distinct molecular subset of melanomas with potential sensitivity to MEK inhibition. Clin Cancer Res. 2013;19:6696-702.

19. Hutchinson KE, Ross JS, Stephens PJ, Miller VA, Sosman JA, Pao W. Melanoma BRAF fusions-response. Clin Cancer Res. 2014;20:6632.

20. Newman S, Pappo A, Raimondi S, Zhang J, Barnhill R, Bahrami A. Pathologic characteristics of Spitz melanoma with MAP3K8 fusion or truncation in a pediatric cohort. Am J Surg Pathol. 2019;43:1631-7.

21. Kim KB, Semrad T, Schrock AB, Ali SM, Ross JS, Singer M. et al. Significant clinical response to a MEK inhibitor therapy in a patient with metastatic melanoma harboring an RAF1 fusion. JCO Precis Oncol. 2018;2:1-6.

22. Frampton GM, Fichtenholtz A, Otto GA, Wang K, Downing SR, $\mathrm{He} \mathrm{J}$, et al. Development and validation of a clinical cancer genomic profiling test based on massively parallel DNA sequencing. Nat Biotechnol. 2013;31:1023-31.

23. Sun JX, He Y, Sanford E, Montesion M, Frampton GM, Vignot S, et al. A computational approach to distinguish somatic vs. germline origin of genomic alterations from deep sequencing of cancer specimens without a matched normal. PLoS Pathog. 2018;14:e1005965.

24. Chalmers ZR, Connelly CF, Fabrizio D, Gay L, Ali SM, Ennis R, et al. Analysis of 100,000 human cancer genomes reveals the landscape of tumor mutational burden. Genome Med. 2017;9:34.

25. Trabucco SE, Gowen K, Maund SL, Sanford E, Fabrizio DA, Hall MJ, et al. A novel next-generation sequencing approach to detecting microsatellite instability and pan-tumor characterization of 1000 microsatellite instability-high cases in 67,000 patient samples. J Mol Diagn. 2019;21:1053-66.

26. Zehir A, Benayed R, Shah RH, Syed A, Middha S, Kim HR, et al. Mutational landscape of metastatic cancer revealed from prospective clinical sequencing of 10,000 patients. Nat Med. 2017;23:703-13.

27. Alexandrov LB, Nik-Zainal S, Wedge DC, Aparicio SAJR, Behjati S, Biankin AV, et al. Signatures of mutational processes in human cancer. Nature. 2013;500:415-21. 
28. Elder DE, Barnhill RL, Bastian BC, Cook MG, de la Fouchardiere A, Gerami P, et al. Melanocytic Tumours. In: Elder D, Massi D, Scolyer R, et al., editors. World Health Organization 2018 Classification of Skin Tumours. Lyon, France: International Agency for Research on Cancer, 2018. p. 65-77.

29. Stransky N, Cerami E, Schalm S, Kim JL, Lengauer C. The landscape of kinase fusions in cancer. Nat Commun. 2014;5:4846.

30. Hayward NK, Wilmott JS, Waddell N, Johansson PA, Field MA, Nones K, et al. Whole-genome landscapes of major melanoma subtypes. Nature. 2017;545:175-80.

31. Martins da Silva V, Martinez-Barrios E, Tell-Martí G, Dabad M, Carrera C, Aguilera P, et al. Genetic abnormalities in large to giant congenital nevi: beyond NRAS mutations. J Invest Dermatol. 2019;139:900-8.

32. Baltres A, Salhi A, Houlier A, Pissaloux D, Tirode F, Haddad V, et al. Malignant melanoma with areas of rhabdomyosarcomatous differentiation arising in a giant congenital nevus with RAF1 gene fusion. Pigment Cell Melanoma Res. 2019;32:708-13.

33. Jones DTW, Kocialkowski S, Liu L, Pearson DM, Ichimura K, Collins VP. Oncogenic RAF1 rearrangement and a novel BRAF mutation as alternatives to KIAA1549:BRAF fusion in activating the MAPK pathway in pilocytic astrocytoma. Oncogene. 2009;28:2119-23.
34. Jain P, Fierst TM, Han HJ, Smith TE, Vakil A, Storm PB, et al. CRAF gene fusions in pediatric low-grade gliomas define a distinct drug response based on dimerization profiles. Oncogene. 2017;36:6348-58.

35. Donati M, Kastnerova L, Martinek P, Grossmann P, Sticová E, Hadravský L, et al. Spitz tumors with ROS1 fusions: A clinicopathological study of 6 cases, including FISH for chromosomal copy number alterations and mutation analysis using next-generation sequencing. Am J Dermatopathol. 2019; 42:92-102

36. Busam KJ, Kutzner H, Cerroni L, Wiesner T. Clinical and pathologic findings of Spitz nevi and atypical Spitz tumors with ALK fusions. Am J Surg Pathol. 2014;38:925-33.

37. Akbani R, Akdemir KC, Aksoy BA, Albert M, Ally A, Amin SB, et al. Genomic classification of cutaneous melanoma. Cell. 2015;161:1681-96.

38. Cutler RE, Stephens RM, Saracino MR, Morrison DK. Autoregulation of the Raf-1 serine/threonine kinase. Proc Natl Acad Sci USA. 1998;95:9214-9.

39. Lee S, Barnhill RL, Dummer R, Dalton J, Wu J, Pappo A, et al. TERT promoter mutations are predictive of aggressive clinical behavior in patients with Spitzoid melanocytic neoplasms. Sci Rep. 2015;5:1-7. 